M. Hashimoto

Nagoya Math. J.

Vol. 118 (1990), 203-216

\title{
DETERMINANTAL IDEALS WITHOUT MINIMAL FREE RESOLUTIONS
}

\author{
MITSUYASU HASHIMOTO
}

\section{Introduction}

Let $R$ be a Noetherian commutative ring with unit element, and $x_{i j}$ be variables with $1 \leq i \leq m$ and $1 \leq j \leq n$. Let $S=R\left[x_{i j}\right]$ be the polynomial ring over $R$, and $I_{t}$ be the ideal in $S$, generated by the $t \times t$ minors of the generic matrix $\left(x_{i j}\right) \in M_{m, n}(S)$. For many years there has been considerable interest in finding a minimal free resolution of $S / I_{t}$, over arbitrary base ring $R$. If we have a minimal free resolution $\boldsymbol{P}$. over $R=Z$, the ring of integers, then $R^{\prime} \otimes_{Z} P$. is a resolution of $S / I_{t}$ over the base ring $R^{\prime}$. When does $S / I_{t}$ have a minimal free resolution over $Z$, then?

The resolution over $Z$ has been found in the case $t=\min (m, n)$ (Eagon-Northcott complex, [3]) and in the case $t=\min (m, n)-1$ (AkinBuchsbaum-Weyman complex, [1]). Of course, in the case $t=1$, we have the resolution of $S / I_{t}$, namely, the Koszul complex. Recently, we proved that $S / I_{t}$ has a minimal free resolution over $Z$ in the case $m=n=t+2$ [5]. But our proof consists in showing that the Betti numbers of $S / I_{t}$ are independent of the characteristic of the ground field, so it does not provide an explicit construction of a resolution.

In this paper, we prove that $S / I_{t}$ does not have any minimal free resolutions, if $R$ is the ring of integers $Z$, and if $2 \leq t \leq \min (m, n)-3$, as we announced in [5]. The third Betti number of $S / I_{t}$ is independent of the characteristic, if $t=1$ or $t \geq \min (m, n)-2$ ([5]). To the contrary, it depends on the characteristic if $2 \leq t \leq \min (m, n)-3$. If the characteristic is 3 , then the Betti number gets larger than the characteristic zero case.

The author would like to thank Professor H. Matsumura for his valuable advice and encouragement. Special thanks are also due to

Received May 24, 1989. 
Doctor J. Nishimura. This work was carried out under his conscientious and patient instruction at Kyoto University. Special thanks are also due to Doctor Kazuhiko Kurano, one of the best friends of mine, for much valuable discussion about this topic. The author would like to appreciate the advice of Professor D. A. Buchsbaum. He read the former version of this paper and pointed out some mathematical and grammatical mistakes. Finally, the author expresses his thanks to the referee for his many pieces of valuable advice.

\section{§1. Preliminaries}

On the characteristic free representation theory of $G L$, including the notion of partitions, Schur modules (Schur functors) and Schur complexes, tableaux, and Cauchy formulae, we use the notation, the terminology and the results of [2] and [5] freely. But we shall review some facts on the characteristic free representation theory of $G L$, which will be used later. For the details, see [2] and [5].

Let $R$ be a commutative ring with unit, and $\alpha: 0 \rightarrow G \stackrel{\Downarrow}{\rightarrow} F \stackrel{\varphi}{\rightarrow} E \rightarrow 0$ be a finite free complex of length two. We define the symmetric algebra of $\alpha$, to be the tensor product: $S \alpha=S E \otimes \wedge F \otimes D G$. S $\alpha$ has a structure of a graded bialgebra over $R$, with an appropriate anticommutative structure. Moreover, $S \alpha$ has a structure of a chain complex. We define the boundary map $\partial^{S \alpha}$ to be the sum, $\partial^{S \varphi} \otimes 1_{D G} \pm 1_{S E} \otimes \partial^{\wedge \psi}$. The multiplication and the comultiplication of $S \alpha$ are chain maps (see [5, chapter I, $\S 2]$ ).

Let $\varphi: F_{1} \rightarrow F_{0}$ and $\psi: G_{1} \rightarrow G_{0}$ be two morphisms of finite free modules, and $k$ be a nonnegative integer. There is a unique universal natural transformation $\theta_{k}$, which makes the following diagram commutative;

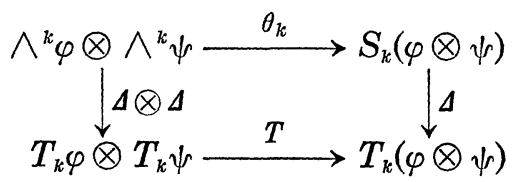

where $\Delta$ 's in the diagram are appropriate diagonalizations, and the $T$ in the diagram is an appropriate twisting. We define $\theta: \wedge \varphi \otimes \wedge \psi \rightarrow S(\varphi \otimes \psi)$ given by $\theta=\theta_{k}$ on $\wedge^{k} \varphi \otimes \wedge^{k} \psi$, and $\theta=0$ on $\wedge^{i} \varphi \otimes \wedge^{j} \varphi$ if $i \neq j$. The natural transformation $\theta$ is the composite map;

$$
\wedge \varphi \otimes \wedge \psi=\wedge F_{0} \otimes D F_{1} \otimes \wedge G_{0} \otimes D G_{1}
$$




$$
\begin{aligned}
& \stackrel{\Delta}{\longrightarrow} \wedge F_{0} \otimes \wedge F_{0} \otimes D F_{1} \otimes D F_{1} \otimes \wedge G_{0} \otimes \wedge G_{0} \otimes D G_{1} \otimes D G_{1} \\
& \stackrel{T}{\longrightarrow} \wedge F_{0} \otimes \wedge G_{0} \otimes D F_{1} \otimes \wedge G_{0} \otimes \wedge F_{0} \otimes D G_{1} \otimes D F_{1} \otimes D G_{1} \\
& \stackrel{\phi^{S} \otimes \phi \wedge \otimes \psi \wedge \otimes \psi^{D}}{\longrightarrow} S\left(F_{0} \otimes G_{0}\right) \otimes \wedge\left(F_{1} \otimes G_{0}\right) \otimes \wedge\left(F_{0} \otimes G_{1}\right) \otimes D_{1}\left(F_{1} \otimes G_{1}\right) \\
& \stackrel{\simeq}{\longrightarrow} S\left(F_{0} \otimes G_{0}\right) \otimes \wedge\left(F_{1} \otimes G_{0} \oplus F_{0} \otimes G_{1}\right) \otimes D\left(F_{1} \otimes G_{1}\right)=S(\varphi \otimes \psi)
\end{aligned}
$$

where $\Delta$ is the diagonalization, $T$ is an appropriate twisting. $\phi^{S}, \phi^{\wedge}, \psi^{\wedge}$, and $\psi^{D}$ are the unique universal natural transformations determined as follows. We define $\phi_{k}^{S}(F, G): \wedge^{k} F \otimes \wedge^{k} G \rightarrow S_{k}(F \otimes G)$ for any nonnegative integer $k$ to be the unique universal natural transformation which makes the following diagram commutative.

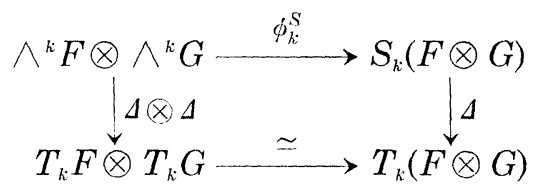

We define $\phi^{S}=\phi_{k}^{S}$ on $\wedge^{k} F \otimes \wedge^{k} G$ and $\phi^{S}=0$ on $\wedge^{i} F \otimes \wedge^{j} G$ if $i \neq j$. Thus $\phi^{S}$ is a natural transformation which maps $\wedge F \otimes \wedge G$ to $S(F \otimes G)$. The definitions of $\phi^{\wedge}, \psi^{\wedge}$, and $\psi^{D}$ are quite similar (see [5, chapter III]). Note that $\phi_{k}^{S}$ is given by

$$
\phi_{k}^{S}\left(f_{1} \wedge \cdots \wedge f_{k} \otimes g_{1} \wedge \cdots \wedge g_{k}\right)=(-1)^{k(k-1) / 2} \operatorname{det}\left(f_{i} \otimes g_{j}\right)_{1 \leq i, j \leq k}
$$

for $f_{1}, \cdots, f_{k} \in F$ and $g_{1}, \cdots, g_{k} \in G$. Since the diagram $(*)$ commutes, $\theta_{k}$ is a chain map.

For a partition $\lambda$ with $\lg (\lambda)=q$ and $|\lambda|=r$, we define $\theta_{\lambda}: \wedge_{\lambda} \varphi \otimes \wedge_{\lambda} \psi$ $\rightarrow S_{r}(\varphi \otimes \psi)$ to be the composite map;

$$
\begin{aligned}
\wedge_{\lambda} \varphi \otimes \wedge_{\lambda} \psi & =\wedge^{\lambda_{1}} \varphi \otimes \cdots \otimes \wedge^{\lambda_{q}} \varphi \otimes \wedge^{\lambda_{1}} \psi \otimes \cdots \otimes \wedge^{\lambda_{q}} \psi \\
& \stackrel{T}{\longrightarrow} \wedge^{\lambda_{1}} \varphi \otimes \wedge^{\lambda_{1}} \psi \otimes \cdots \otimes \wedge^{\lambda_{q}} \varphi \otimes \wedge^{\lambda_{q}} \psi \\
& \stackrel{\theta_{\lambda_{1}} \otimes \cdots \otimes \theta_{\lambda_{q}}}{\longrightarrow} S_{\lambda_{1}}(\varphi \otimes \psi) \otimes \cdots \otimes S_{\lambda_{q}}(\varphi \otimes \psi) \stackrel{m}{\longrightarrow} S_{r}(\varphi \otimes \psi)
\end{aligned}
$$

where $T$ is an appropriate twisting, and $m$ is the (iterated) multiplication. We also define:

$$
M_{\lambda}(\theta)=\sum_{\substack{|\mu|=r \\ \mu \geq \lambda}} \operatorname{Im} \theta_{\mu} \quad \text { and } \quad \dot{M}_{\lambda}(\theta)=\sum_{\substack{|\mu|=2 r \\ \mu>\lambda}} \operatorname{Im} \theta_{\mu^{\prime}}
$$

For $r \in N_{0},\left\{M_{\lambda}(\theta)\right\}_{|\lambda|=r}$ gives a filtration of $S_{r}(\varphi \otimes \psi)$.

The Cauchy formula holds for $S(\varphi \otimes \psi)$ via the pairing $\theta$. 
Lemma 1.1 ([5, Proposition III, 2.6]). Let $\lambda \in \Omega_{k}^{+}$and $\mu \in S_{\square}(\lambda)$. The following diagram is commutative.

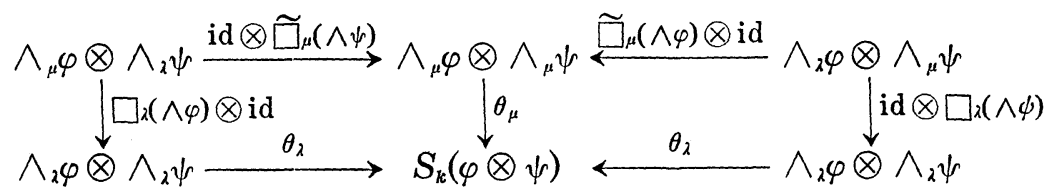

Theorem 1.2 ([5: Theorem III. 2.7]). Let $k \in N_{0}$, and $\varphi: F_{1} \rightarrow F_{0}$ and $\psi: G_{1} \rightarrow G_{0}$ be morphism of finite free $R$-modules. If $\lambda \in \Omega_{\bar{k}}$, then $\theta_{\lambda}$ induces the isomorphism of complexes $\beta_{\lambda}: L_{\lambda} \varphi \otimes L_{\lambda} \psi \rightarrow M^{\lambda}(\theta) / \dot{M}^{\lambda}(\theta)$ which makes the following diagram commutative;

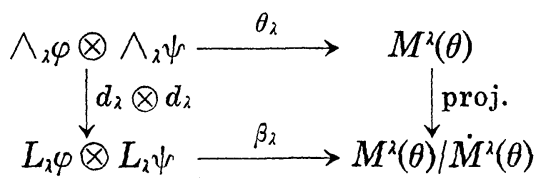

where $L_{\lambda}$ is the Schur complex with respect to the shape $\lambda$. Hence, the associated graded complex of the filtration $\left\{M^{\lambda}(\theta)\right\}_{\lambda \in \Omega_{\bar{k}}}$ is $\sum_{\lambda \in \Omega_{\bar{k}}} L_{\lambda} \varphi \otimes L_{\lambda} \psi$.

Now we fix positive integers $m, n$, and $t$ with $t \leq \min (m, n)$, and we consider free $R$-modules $F$ and $G$ with $\operatorname{rank} F=m$ and $\operatorname{rank} G=n$. We let $S=S(F \otimes G)$ so that $S$ is isomorphic to the polynomial ring with $m \cdot n$ variables over $R$. We define $I_{t}$ to be the ideal of $S$ generated by Im $\phi_{t}^{S}$ and call $I_{t}$ a determinantal ideal. For $r \in N_{0}$, we denote $S_{r}(F \otimes G)$ by $S_{r}$, and $S_{r} \cap I_{r}$ by $I_{t, r}$. We denote the complex $I_{t} \otimes_{S} S\left(\operatorname{id}_{F \otimes G}\right)$ (resp. $\left.I_{t} \otimes_{S} S\left(\mathrm{id}_{F} \otimes \mathrm{id}_{G}\right)\right)$ by $\mathscr{I}^{t}$ (resp. $\left.\tilde{I}^{t}\right)$. The complex $\mathscr{I}^{t}$ (resp. $\left.\tilde{\mathscr{I}}^{t}\right)$ is a graded $S$-complex so that $\mathscr{I}^{t}$ (resp. $\tilde{I}^{t}$ ) is decomposed into the direct sum; $\mathscr{I}^{t}=$ $\sum_{r \in N_{0}} \mathscr{I}^{t, r} \quad$ (resp. $\left.\tilde{I}^{t}=\sum_{r \in N_{0}} \tilde{I}^{t, r}\right)$. Since $S\left(\mathrm{id}_{F \otimes G}\right)=S \otimes \wedge(F \otimes G)$ is a graded minimal free resolution of $R=S / I_{1}, H_{i}\left(\mathscr{I}^{t, r}\right)$ is the degree $r$ component $\left[\operatorname{Tor}_{i}^{S}\left(I_{t}, S / I_{1}\right)\right]_{r}$ of the graded $S$-module $\operatorname{Tor}_{i}^{S}\left(I_{t}, S / I_{1}\right)$ for any $i \geq 0$ and $r \geq 0$. On the other hand, we have an isomorphism $H_{i}\left(\mathscr{I}^{t, r}\right) \simeq$ $H_{i}\left(\tilde{I}^{t, r}\right)$ for any $i$ and $r$ [5, Lemma IV. 1.4]. In case $R=K$ is a field of characteristic $p$, we denote $\operatorname{dim}_{K}\left[\operatorname{Tor}_{i}^{S}\left(S / I_{t}, S / I_{1}\right)\right]_{r}$, which is invariant under an extention of the base field $K$, by $\beta_{i, r}^{p}$. We have the following lemma.

LEMMA 1.3. There is a minimal free resolution of $S / I_{t}$ in the case $R=Z$, if and only if $\beta_{i+1, r}^{p}=\operatorname{rank} H_{i}\left(\tilde{I}^{t, r}\right)$ is independent of the characteristic $p$ of the base field $R=K$ for any $i \geq 0$.

For the proof of the lemma, see [9, Proposition 2 of chapter 4] or 
[5, Proposition II. 3.4].

Now we shall prepare some additional notation. We define $\pi: \mathrm{id}_{F} \otimes \mathrm{id}_{G}$ $\rightarrow \mathrm{id}_{F \otimes G}$ and $\iota: \mathrm{id}_{F \otimes G} \rightarrow \mathrm{id}_{F} \otimes \mathrm{id}_{G}$ to be the morphisms of complexes given by:

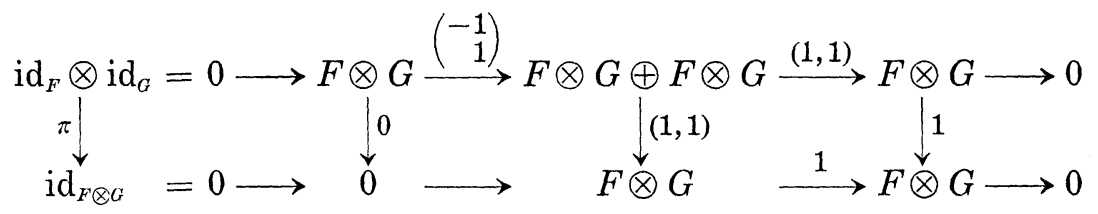

and

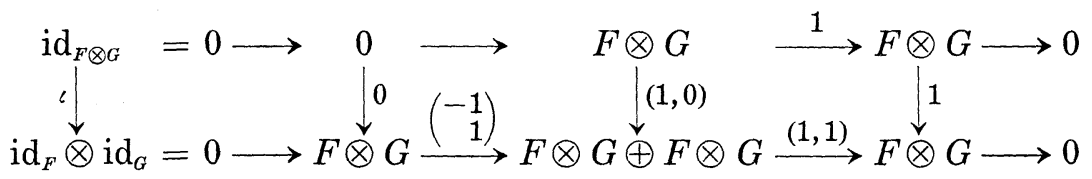

It is easy to see that $\pi \circ \iota=\mathrm{id}_{\mathrm{id}_{F \otimes G}}$. For $r \in N_{0}, S_{r} \pi$ maps $\tilde{I}^{t, r}$ onto $\mathscr{I}^{t, r}$, and $S_{r^{\iota}}$ maps $\mathscr{I}^{t, r}$ into $\tilde{I}^{t, r}$. Since $H_{*}\left(\tilde{I}^{t, r}\right) \simeq H_{*}\left(\mathscr{I}^{t, r}\right)$ and $H_{*}\left(S_{r} \pi\right) \circ$ $H_{*}\left(S_{r} \iota\right)=\mathrm{id}, H_{*}\left(S_{r} \pi\right)$ gives an explicit isomorphism between them. We define $\alpha^{r}: \wedge^{r} \mathrm{id}_{F} \otimes \wedge^{r} \mathrm{id}_{G} \rightarrow S_{r}\left(\mathrm{id}_{F \otimes G}\right)$ to be the composition $S_{r} \pi \circ \theta_{r}$ for $r \in N_{0}$. It is clear that $\alpha^{r}$ maps $L_{k}^{t,(r)}=\sum_{i+j=k} L_{i, j}^{t,(r)}$ to $\mathscr{I}_{k}^{t, r}$ for $t, k \in N_{0}$ (for the definition of $L_{i, j}^{t, \lambda}$ ( $\lambda$ a partition), see [5, Definition IV. 1.5]). Note that $L^{t,(r)}$ is nothing but the complex $\left\{\boldsymbol{U}^{t}(F, G), \partial^{t}\right\}$ defined in [1, Definition 3.7]. The map $\alpha^{r}$ coincides with the map defined in [1, Remark 3.19]. If $R$ contains $\boldsymbol{Q}$, then $\alpha_{k}^{t, t+k}: L_{k}^{t,(t+k)} \rightarrow Z_{k+1}^{t, t}=\partial_{k}^{-1}\left(\mathscr{I}_{k-1}^{t+1, t+k}\right)$ is surjective, but this is not true in general (see section 3 ).

We fix ordered bases $X=X_{0} \cup X_{1}$ of $\mathrm{id}_{F}: F_{1} \rightarrow F_{0}$ and $Y=Y_{0} \cup Y_{1}$ of $\operatorname{id}_{G}: G_{1} \rightarrow G_{0}$, where $X_{0}=\left\{x_{1}<\cdots<x_{m}\right\}, X_{1}=\left\{x_{1}^{\prime}<\cdots<x_{m}^{\prime}\right\}, \quad Y_{0}=\left\{y_{1}<\right.$ $\left.\cdots<y_{n}\right\}$ and $Y_{1}=\left\{y_{1}^{\prime}<\cdots<y_{n}^{\prime}\right\}$ are bases of $F_{0}, F_{1}, G_{0}$ and $G_{1}$, respectively. The ordering is given by $X_{0}<X_{1}$ and $Y_{0}<Y_{1}$. For simplicity of notation, we may denote $x_{i}$ and $y_{i}$ by $i$, and $x_{i}^{\prime}$ and $y_{i}^{\prime}$ by $i^{\prime}$, if there is no danger of confusion.

For a tableau $S \in \operatorname{Tab}_{\lambda / \mu}(X)$ and subsets $I \subset X$ and $N \subset N$, we denote $\#\left\{(i, j) \in \Delta_{\lambda / \mu} \mid i \in N\right.$ and $\left.T(i, j) \in I\right\}$ by $n_{N}(T, I)$. In this notation, an element $x \in X$ (resp. $i \in N$ ) may stand for the singleton $\{x\}$ (resp. $\{i\}$ ). We denote $n_{i}\left(S, X_{1}\right)$ by $n_{i}(S)$, and $n_{N}\left(S, X_{1}\right)$ by $n(S)$. We will use a similar convention for a tableau $T \in \operatorname{Tab}_{\lambda / \mu}(Y)$.

Let $\lambda \in \Omega^{-}, S \in \operatorname{Tab}_{\lambda} X$, and $T \in \operatorname{Tab}_{\lambda} Y$. We use the bitableau notation as in [2]. We denote $\theta_{\lambda}(S \otimes T)$ by $(S \mid T)$. More generally, we will denote 
$\theta_{\lambda}(a \otimes b)$ by $(a \mid b)$ for $a \in \wedge_{\lambda} \mathrm{id}_{F^{\prime}}$ and $b \in \wedge_{\lambda} \mathrm{id}_{G}$. The set of tableaux, $\left\{S \in \operatorname{Tab}_{\lambda} X \mid S\right.$ is row-standard $\left.\bmod X_{1}\right\}$, is denoted by $X_{\lambda}$. The set $Y_{\lambda}$ is defined similarly.

Let $R=K$ be an infinite field, and $M$ be a polynomial representation of $G L(F)$ (i.e., $M$ be a $K[\operatorname{End}(F)]$-module with $\operatorname{dim}_{K} M<\infty$, and the representation map $\rho$ : End $(F) \rightarrow$ End $(M)$ be a regular morphism). We identify End $(F)$ with $M_{n}(K)$ via the basis $X=\left\{x_{1}, \cdots, x_{m}\right\}$. For a sequence $\alpha=\left(\alpha_{1}, \cdots, \alpha_{m}\right) \in N_{0}^{m}$, we define the subspace $M_{\alpha}$ of $M$ by

$$
M_{\alpha}=\left\{\left.a \in M\right|^{\forall}\left(t_{1}, \cdots, t_{m}\right) \in K^{m} \rho\left(t_{1} \oplus \cdots \oplus t_{m}\right) \cdot a=t_{1}^{\alpha_{1}} \cdots t_{m}^{\alpha_{m}} \cdot a\right\}
$$

where $t_{1} \oplus \cdots \oplus t_{m}$ is a diagonal matrix whose $(i, i)$ content is $t_{i}$. We call $M_{\alpha}$ the $\alpha$-weight submodule of $M$, and $\alpha$ its weight. The representation $M$ is decomposed into the direct sum of $M_{\alpha}$. Any morphism of polynomial representations of $G L(F)$ preserves weight. So any chain complex of polynomial representations of $G L(F)$, say $\boldsymbol{P}$, is decomposed into the direct sum; $\boldsymbol{P}=\sum_{\alpha} \boldsymbol{P}_{\alpha}$.

We will consider complexes of polynomial representations of $G L(F)$ $\times G L(G)$ in section 3. Such a complex, say $C$, is decomposed into the direct sum of biweight subcomplexes $C_{\alpha}$ corresponding to the biweight $\alpha=(\alpha(F) ; \alpha(G))$. For example, the biweight $\left(\alpha_{1}, \cdots, \alpha_{m} ; \beta_{1}, \cdots, \beta_{n}\right)$ submodule of $S_{k}\left(\mathrm{id}_{F} \otimes \mathrm{id}_{G}\right)$ is generated by:

$$
\begin{aligned}
& \left\{(S \mid T) \mid{ }^{\exists} \lambda \in \Omega_{k}^{-}, S \in X_{\lambda}, T \in Y_{\lambda}, \forall i(1 \leq i \leq m)\right. \\
& \left.n_{N}\left(S,\left\{x_{i}, x_{i}^{\prime}\right\}\right)=\alpha_{i}, \quad \forall j(1 \leq j \leq n) n_{N}\left(T,\left\{y_{j}, y_{j}^{\prime}\right\}\right)=\beta_{j}\right\}
\end{aligned}
$$

Any universally free functor $L$ on $F$ and $G$ that we will consider will always be a polynomial functor. So $L(F, G)$ is a polynomial representation of $G L(F) \times G L(G)$.

\section{§2. The filtration of $\tilde{I}^{t, r}$}

We have calculated $\beta_{3}^{p}$ in the case $t \geq \min (m, n)-2$, in [5], using the natural filtration $\left\{M^{t, \lambda}\right\}_{\lambda \in \Omega_{r}^{-}}$of $\tilde{\mathscr{I}}^{t, r}$. We can associate with this filtration the usual spectral sequence whose $E^{1}$-term is $E_{*}^{1, t, \lambda}=H_{*}\left(M^{t, \lambda} / \dot{M}^{t, \lambda}\right)$. We use the following facts on the homology of the associated graded complex of this filtration.

Proposition 2.1. Let $m, n, r$ and $t$ be positive integers with $\min (m, n)$ $\geq t$, and $\lambda \in \Omega_{r}^{-}$. Then we have:

(1) $E_{1}^{1, t, \lambda}=0$, except for the case $\lambda=(t+1)$. In particular, $H_{1}\left(\mathscr{I}^{t, r}\right)$ 
$=0$ except for the case $r=t+1$.

(2) $E_{2}^{1, t, \lambda}=0$, except for the following three cases.

(i) $\lambda=(t+2)$

(ii) $r=2 t+1, \lambda=(t+1, t), 1 /(t+1) \notin R$, and $\min (m, n) \geq r$

(iii) $t<r \leq 2 t, \lambda=(t, r-t), 1 /(r-t) \notin R$, and $\min (m, n) \geq r$

(3) If the following two conditions hold, then $E_{3}^{1, t, \lambda}=0$.

(i) $\lambda_{1}=t$ or $\lambda_{2}<t$

(ii) $\lg (\lambda) \geq 3$, or equivalently, $\lambda_{3} \neq 0$

Proof. (3) is [5, Proposition IV. 3.1]. (2) is a little stronger than [5, Proposition IV. 2.3]. We have to show that $E_{2}^{1, t, \lambda}=0$ if $\lambda \neq(t+2)$ and if $r-t$ is invertible in $R$.

We use the same spectral sequence argument used in the proof of [5, Proposition IV. 2.3]. By Lemma IV. 2.4 and Lemma IV. 2.7 of [5], we have only to show that $E_{1,1}^{2}=H_{1}^{G}\left(H_{1}^{F}\left(M_{*, *}^{t, 2} / \dot{M}_{*, *}^{t, \lambda}\right)\right)=0$.

First we consider the case $t<r \leq 2 t$, and $\lambda=(t, r-t)$. In this case the same argument as in the proof of [5, Lemma IV. 2.8] works. In fact, any element of $E_{1,1}^{2}$ is represented by $A=\sum_{S, T} c_{S, T}(S \mid T)$, where $S$ is standard $\bmod X_{1}, T$ is standard $\bmod Y_{1}$, and $n_{1}(S)=n_{1}(T)=0$. So we can write $\sum_{S} c_{S, T} \partial_{F}^{\lambda} S=\sum_{\mu \in S_{\square^{(\lambda)}}} \square_{\lambda}^{\mu}\left(a_{\mu}^{T}\right)$, where $a_{\mu}^{T} \in \wedge_{\mu} F$. But since $\square_{\lambda}^{(r)}\left(a_{(r)}^{T}\right)=$ $1 /(r-t) \square_{\lambda}^{(r-1,1)}\left(\square_{(r-1,1)}^{(r)}\left(a_{(r)}^{T}\right)\right)$, we may assume that $a_{(r)}^{T}=0$, after replacing $a_{(r-1,1)}^{T}$ by $a_{(r-1,1)}^{T}+1 /(r-t) \square_{(r-1,1)}^{(r)}\left(a_{(r)}^{T}\right)$. So this case is clear.

We consider the case $\lambda=(t+1, t)$. Any element of $E_{1,1}^{2}$ is represented by $A=\sum_{S, T} c_{s, T}(S \mid T)$, where $S \in X_{\lambda}, T \in Y_{\lambda}, S$ is standard $\bmod X_{1}, T$ is standard $\bmod Y_{1}$, and $n(S)=n(T)=1$. We claim that for each pair $(S, T)$, which appears in the sum with $n_{1}(S)=n_{1}(T)=1$, it holds

$$
(S \mid T) \in \theta_{\lambda}\left(L_{1,1}^{t, \lambda, 1}\right)+\dot{M}_{1,1}^{t, \lambda}+\partial_{F}\left(M_{2,1}^{t, \lambda}\right)+\partial_{G}\left(M_{1,2}^{t, \lambda}\right) .
$$

If the claim is true, we may assume that $A \in \theta_{\lambda}\left(L_{1,1}^{t, \lambda, 1}\right)+\partial_{G}\left(M_{1,2}^{t, \lambda}\right)$. So we can write $A=A^{\prime}+\partial_{G} B$ with $A^{\prime} \in \theta_{\lambda}\left(L_{1,1}^{t, \lambda, 1}\right)$ and $B \in M_{1,2}^{t, \lambda}$. It is easy to see that there exists some $B^{\prime} \in \theta_{2}\left(L_{1,2}^{t, \lambda, 1}\right)$ such that $\partial_{F}\left(B-B^{\prime}\right) \in \dot{M}_{0,2}^{t, \lambda}$ (see the proof of [5, Lemma IV. 2.4]). Replacing $A=A^{\prime}+\partial_{G} B$ by $A^{\prime}+\partial_{G} B^{\prime}$, we may assume $A \in \theta_{\lambda}\left(L_{1,1}^{t, \lambda, 1}\right)$. So the proof of [5, Lemma IV. 2.8] is still valid by [5, Lemma IV. 2.6], and it suffices to prove the claim.

We shall prove the claim.

We put;

$$
S=\begin{aligned}
& a_{1} \cdots a_{t} a_{t+1}^{\prime} \\
& b_{1} \cdots b_{t}
\end{aligned} \text { and } T=\begin{aligned}
& \alpha_{1} \cdots \alpha_{t} \alpha_{t+1}^{\prime} \\
& \beta_{1} \cdots \beta_{t}
\end{aligned}
$$


where $a_{i}$ and $b_{j}$ are elements of $X_{0}$, and $\alpha_{i}$ and $\beta_{j}$ are elements of $Y_{0}$. We may assume that $\alpha_{i}$ ans $\beta_{j}$ are all distinct (if not, then the claim is (essentially) proved in [5, Lemma IV. 2.5]). If we set;

$$
S^{\prime}=\begin{aligned}
& a_{1} \cdots a_{t} a_{t+1} \\
& b_{1} \cdots b_{t}^{\prime}
\end{aligned}
$$

then we have

$$
\begin{aligned}
& (S \mid T)-\left(S^{\prime} \mid T\right)=\frac{1}{t+1} \cdot \partial_{G}\left(S \mid \sum_{j=1}^{t}(-1)^{t-j} \begin{array}{c}
\alpha_{1} \cdots \alpha_{t} \alpha_{j}^{\prime} \alpha_{t+1}^{\prime} \\
\beta_{1} \cdots \beta_{t}
\end{array}\right) \\
& +\frac{1}{t+1} \cdot \partial_{G}\left(S^{\prime} \mid t \cdot \begin{array}{c}
\alpha_{1} \cdots \alpha_{t} \alpha_{t+1}^{\prime} \\
\beta_{1} \cdots \beta_{t}^{\prime}
\end{array} \sum_{j=1}^{t}(-1)^{t-j} \begin{array}{c}
\alpha_{1} \cdots \alpha_{t} \\
\beta_{1} \cdots \beta_{t-1} \beta_{t}^{\prime}
\end{array}\right)
\end{aligned}
$$

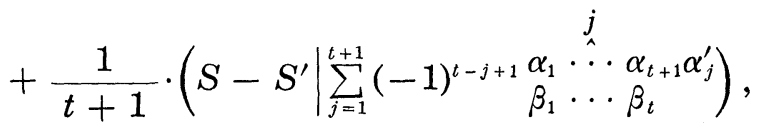

where each symbol $j$ indicates the deletion of the $j$-th member in the sequence. Hence, it suffices to show that the element

$$
C=\frac{1}{t+1} \cdot\left(S-S^{\prime} \mid \sum_{j=1}^{t+1}(-1)^{t-j+1} \begin{array}{c}
\alpha_{1} \cdots \\
\beta_{1} \cdots \alpha_{t+1} \alpha_{j}^{\prime}
\end{array}\right)
$$

is contained in $\partial_{F}\left(M_{2,1}^{t, \lambda}\right)$. We shall calculate $C$. If we put

$$
U=\sum_{j=1}^{t+1}(-1)^{t-j+1} \begin{gathered}
\alpha_{1} \cdots \alpha_{t+1} \\
\beta_{1} \cdots \beta_{t} \\
\alpha_{j}^{\prime}
\end{gathered} \in\left[\wedge_{(t, t, 1)} \mathrm{id}_{G}\right]_{1},
$$

then using Lemma 1.1, we have

$$
\begin{aligned}
& C=\frac{1}{t+1} \cdot\left(\begin{array}{l}
a_{1} \cdots a_{t} \\
b_{1} \cdots b_{t} \\
a_{t+1}^{\prime}
\end{array}+\sum_{j=1}^{t}(-1)^{t+1-j} \begin{array}{c}
a_{1} \cdots a_{t} a_{t+1}^{\prime} \\
b_{1} \cdots \cdots \\
a_{j}
\end{array} \mid U\right)
\end{aligned}
$$

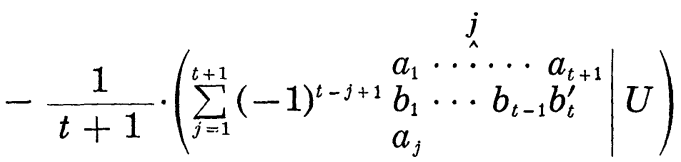

$$
\begin{aligned}
& =\frac{1}{t+1} \cdot \partial\left(\sum_{j=1}^{t}(-1)^{t-j} \begin{array}{c}
a_{1} \cdot \hat{\lambda} a_{t} a_{t+1}^{\prime} \\
b_{1} \cdots \cdots b_{t}^{\prime} \\
a_{j}^{\prime}
\end{array}-\sum_{j=1}^{t+1}(-1)^{t-j} \begin{array}{c}
a_{1} \cdots \hat{\lambda} \\
b_{1} \cdots b_{t-1} b_{t+1}^{\prime} \\
a_{j}^{\prime}
\end{array} \mid U\right)+D,
\end{aligned}
$$

where $D \in M_{2,0}^{t,(t, t, 1)}$ is of the form $D=\left(V \mid \partial_{G} U\right)$. Since 


$$
\partial_{G} U=\sum_{j=1}^{t+1}(-1)^{t-\jmath+1} \begin{gathered}
\alpha_{1} \cdots \\
\beta_{1} \cdots \\
\alpha_{j}
\end{gathered}
$$

and

$$
\sum_{j=1}^{t+1}(-1)^{t-\jmath+1} \alpha_{1} \wedge . \wedge_{\wedge}^{j} \wedge \alpha_{t+1} \otimes \alpha_{j}=\Delta\left(\alpha_{1} \wedge \cdots \wedge \alpha_{t+1}\right)
$$

it holds that $D \in M_{2,0}^{t, \lambda}$. Hence,

$$
\frac{1}{t+1} \cdot \partial\left(\sum_{j=1}^{t}(-1)^{t-j} \begin{array}{c}
a_{1} \cdots a_{t} a_{t+1}^{\prime} \\
b_{1} \cdots \cdots b_{t} \\
a_{j}^{\prime}
\end{array}-\sum_{j=1}^{t+1}(-1)^{t-j} \begin{array}{c}
a_{1} \cdots \hat{\lambda} \\
b_{1}^{\prime} \cdots b_{t-1} b_{t}^{\prime} \\
a_{j}^{\prime}
\end{array} \mid U\right)+D
$$

is a cycle of $M^{t,(t, t, 1)} / M^{t, 2}$. By (3) of this proposition (see also Proposition 2.3 below), $C-D$ is a boundary of $M^{t, \lambda}$ so that $C \in \partial_{F}\left(M_{2,1}^{t, \lambda}\right)$. This proves our claim, so we have completed the proof of (2).

(1) can be proved quite similarly to (2), and so we omit the proof.

Remark 2.2. From (1) of (2.1), we can conclude that $\beta_{2, r}=0$, unless $r=t+1$. Furthermore, we can see that $\boldsymbol{X}_{2}^{t}=H_{1}\left(\mathscr{J}^{t, t+1}\right)=E_{1}^{1, t,(t+1)}$ is a homomorphic image of $H_{1}\left(L^{t,(t+1)}\right)$ by the morphism $H_{1}\left(\alpha^{t, t+1}\right)$. Using this fact, it is not difficult to see that $X_{2}^{t}$ is generated by the elements of the following form;

$$
\partial\left(i_{2} \cdots i_{t} i_{1}^{\prime} i_{t+1}^{\prime} \mid j_{1} \cdots j_{t+1}\right) \text { with } i_{i_{t+1}}^{i_{1} \cdots i_{t}} \text { and } j_{1} \cdots j_{t+1} \text { are both standard }
$$

and

$$
\partial\left(i_{2} \cdots i_{t+1} i_{1}^{\prime} \mid j_{1} \cdots j_{t} j_{t+1}^{\prime}\right) \text { with } i_{1} \cdots i_{t+1} \text { and }{\underset{j}{t+1}}_{\substack{j_{1} \\ j_{t}}} j_{t} \text { are both standard }
$$

where $\partial$ is the boundary map of $S\left(\mathrm{id}_{F} \otimes \mathrm{id}_{G}\right)$. Since

$$
\operatorname{rank} X_{2}^{t}=\operatorname{rank}\left[L_{(t, 1)} F \otimes \wedge^{t+1} G \oplus \wedge^{t+1} F \otimes L_{(t, 1)} G\right],
$$

these elements are a free basis of $\boldsymbol{X}_{2}^{t}$.

These facts were first proved essentially by Kurano [6].

Proposition 2.3. We let $\lambda_{0}=(3,2)$ if $t=2$, and $\lambda_{0}=(t, 3)$ if $t \geq 3$. Then $E_{2}^{1, t, \lambda_{0}} \simeq E_{2}^{\infty, t, \lambda_{0}}$. In particular, if we have $E_{2}^{1, t, \lambda_{0}} \neq 0$, then $\beta_{3, t+3} \neq 0$.

Proof. If $\mu$ is a partition of weight $t+3$ with $\mu<\lambda_{0}$ in the lexicographic order, then $\mu$ satisfies the conditions (i) and (ii) of (3) in Pro- 
position 2.1 , so that $E_{3}^{1, t, \mu}=0$. We have $E_{1}^{1, t, \nu}=0$ for any partition $\nu$ of weight $t+3$ by (1) of the proposition. With these facts and the standard spectral sequence argument, it is easy to see that $E_{2}^{1, t, \lambda_{0}} \simeq E_{2}^{\infty, t, \lambda_{0}}$. The second assertion is now clear and the proof is complete.

By Lascoux's resolution [7], we know that $\beta_{3, t+3}^{0}=0$. Furthermore, we can see that $\beta_{3, t+3}^{p}=0$ if $p$ is a prime number with $p \neq 3$ by (2) of Proposition 2.1. We shall show that $\beta_{3, t+3}^{3} \neq 0$, if $2 \leq t \leq \min (m, n)-3$.

\section{§3. The main result}

This section is devoted to prove the next theorem.

TheOREM 3.1. Let $m, n$ and $t$ be positive integers with $2 \leq t \leq \min (m, n)$ - 3. Then the third Betti number $\beta_{3}$ of $S / I_{t}$ depends on the characteristic. In this case, $S / I_{t}$ does not have any minimal free resolutions over $Z$.

Proof. By the argument in section 2 and Lemma 1.3, we see that it sufficies to show that $E_{2}^{1, t, \lambda_{0}} \neq 0$ when $R$ is an infinite field $K$ of characteristic three, where $\lambda_{0}$ is the partition defined in Proposition 2.3. Each $M^{t, 2}$ is decomposed into the direct sum of the summands indexed by the bicontents (see section 1). So it is sufficient to show that the biweight

$$
\alpha=(1,1, \cdots, 1,0, \cdots, 0 ; 1,1, \cdots, 1,0, \cdots, 0)
$$

submodule of $E_{2}^{1, t, \lambda_{0}}$ is not zero. We shall show that $E=E_{2, \alpha}^{1, t, \lambda_{0}}=\left[E_{2}^{1,1, \lambda_{0}}\right]_{\alpha}$ is not zero. To this end, we construct a non-zero linear form $h: E \rightarrow K$.

(i) case $1 . \quad t=2$.

First, we construct a linear form $g: L_{1,1, \alpha}^{t, \lambda_{0}} \rightarrow K$. Note that $L_{1,1, \alpha}^{t, \lambda_{0}}=$ $L_{1,1, \alpha}^{t, \lambda_{0}, 1} \oplus L_{1,1, \alpha}^{t, \lambda_{0}, 2}$. It holds that

$$
L_{1,1, \alpha}^{t, \lambda_{0}, 2}=\left[\wedge^{2} F \otimes D_{1} F \otimes \wedge^{2} F\right]_{\alpha(F)} \otimes\left[\wedge^{2} G \otimes D_{1} G \otimes \wedge^{2} G\right]_{\alpha(G)}
$$

where []$_{\alpha(F)}$ and []$_{\alpha(G)}$ indicates the weight $(1,1,1,1,1,0,0, \cdots)$-submodule. Hence, the basis element of $L_{1,1, \alpha}^{t, \lambda_{0}, 2}$ is of the form

$$
S \otimes T={ }_{\sigma 4 \sigma 5}^{\sigma 1 \sigma 2(\sigma 3)^{\prime}} \otimes \underset{\tau 4 \tau 5}{\tau 1 \tau 2(\tau 3)^{\prime}}
$$

with $\sigma, \tau \in \mathbb{S}_{5}$, and $S$ and $T$ both row-standard $\left(\bmod X_{1}\right.$ or $\left.\bmod Y_{1}\right)$. For such a basis element, define $g(S \otimes T)=(-1)^{\sigma \tau}$. We define $g$ to be zero on $L_{1,1, \alpha}^{t, \lambda_{0}, 1}$. This gives the definition of $g$. We shall see that $g$ induces a 
linear form $\bar{g} ; M_{1,1, \alpha}^{t, \lambda_{0}} / \dot{M}_{1,1, \alpha}^{t, \lambda_{0}} \rightarrow K$. To see this, it suffices to prove that $g$ vanishes on

$(\operatorname{Ker} \bar{\theta})$

$$
\begin{aligned}
& =\left[\square_{\lambda_{0}}^{(5)}\left(\wedge^{4} F \otimes D_{1} F\right)+\square_{\lambda_{0}}^{(4,1)}\left(\wedge^{3} F \otimes D_{1} F \otimes \wedge^{1} F\right)\right] \otimes\left[\wedge^{2} G \otimes D_{1} G \otimes \wedge^{2} G\right]_{\alpha(G)} \\
& +\left[\wedge^{2} F \otimes D_{1} F \otimes \wedge^{2} F\right]_{\alpha\left(F^{\prime}\right)} \otimes\left[\square_{\lambda_{0}}^{(5)}\left(\wedge^{4} G \otimes D_{1} G\right)+\square_{\lambda_{0}}^{(4,1)}\left(\wedge^{3} G \otimes D_{1} G \otimes \wedge^{1} G\right)\right] \\
& +(\operatorname{Ker} \bar{\theta}) \cap L_{1,1, \alpha}^{t, \lambda_{0}, 1}
\end{aligned}
$$

where $\bar{\theta}$ is the composite map:

$$
L_{1,1, \alpha}^{t, \lambda_{0}} \stackrel{0_{\lambda_{0}}}{\longrightarrow} M_{1,1, \alpha}^{t, \lambda_{0}} \stackrel{\text { proj. }}{\longrightarrow} M_{1,1, \alpha}^{t, \lambda_{0}} / \dot{M}_{1,1, \alpha}^{t, \lambda_{0}} .
$$

The equation is a consequence of Theorem 1.2. We consider the linear form: $g_{F}:\left[\wedge_{\lambda_{0}} \mathrm{id}_{F}\right]_{1, \alpha\left(F^{\prime}\right)} \rightarrow K$ defined by:

$$
\begin{gathered}
g_{F} \text { is zero on }\left[\wedge^{3} F \otimes \wedge^{1} F \otimes D_{1} F\right]_{\alpha\left(F^{\prime}\right)} \\
g_{F}\left(\begin{array}{lll}
\sigma 1 & \sigma 2 & (\sigma 3)^{\prime} \\
\sigma 4 & \sigma 5
\end{array}\right)=(-1)^{\sigma} \quad \text { for } \sigma \in \mathbb{S}_{5} .
\end{gathered}
$$

The linear form $g_{G}:\left[\wedge_{\lambda_{0}} \mathrm{id}_{G}\right]_{1, \alpha(G)} \rightarrow K$ is defined similarly. It holds that $g=g_{F} \otimes g_{G}$ on $L_{1,1, \alpha}^{t, \lambda_{0}}$. We see that;

$$
\begin{aligned}
& g_{F^{\prime}} \circ \square_{\lambda_{0}}^{(5)}\left(\sigma 1 \quad \sigma 2 \quad \sigma 3 \quad \sigma 4(\sigma 5)^{\prime}\right)=(-1)^{\sigma} \cdot\left(\begin{array}{l}
4 \\
2
\end{array}\right)=0 \\
& g_{F} \circ \square_{\lambda_{0}}^{(4,1)}\left(\begin{array}{llll}
\sigma 1 & \sigma 2 & \sigma 3 & (\sigma 4)^{\prime} \\
\sigma 5 & &
\end{array}\right)=(-1)^{\sigma} \cdot 3=0
\end{aligned}
$$

by a straightforward computation. Hence, $g_{F}$ vanishes on

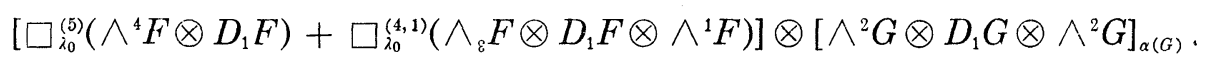

Similar calculation will show that $g_{G}$ vanishes on

$$
\left[\wedge^{2} F \otimes D_{1} F \otimes \wedge^{2} F\right]_{\alpha(F)} \otimes\left[\square_{\lambda_{0}}^{(5)}\left(\wedge^{4} G \otimes D_{1} G\right)+\square_{\lambda_{0}}^{(4,1)}\left(\wedge^{3} G \otimes D_{1} G \otimes \wedge^{1} G\right)\right] .
$$

It is clear that $g$ vanishes on $L_{1,1, \alpha}^{t, \lambda_{0}, 1}$. We conclude that $g$ induces $\bar{g}$. We extend the definition of $\bar{g}$. We define $\bar{g}$ is zero on $M_{2,0, \alpha}^{t, \lambda_{0}} / \dot{M}_{2,0, \alpha}^{t, \lambda_{0}} \oplus M_{0,2, \alpha}^{t, \lambda_{0}} / \dot{M}_{0,2, \alpha}^{t, \lambda_{0}}$ so that $\bar{g}$ is defined over $M_{2, \alpha}^{t, \lambda_{0}} / \dot{M}_{2, \alpha}^{t, \lambda_{0}}$.

Now we shall show that $\bar{g}$ induces $h: E \rightarrow K$. To see this, it is sufficient to show that $\bar{g}$ is zero on $\left[\dot{M}_{2, \alpha}^{t, \lambda_{0}}+B_{2}\left(M_{\alpha}^{t, \lambda_{0}}\right)\right] / \dot{M}_{2, \alpha}^{t, \lambda_{0}}$. To see this, it is sufficient to show that $\bar{g}$ vanishes on

$$
\bar{\theta}\left(\partial_{F}\left(L_{2,1, \alpha}^{t, \lambda_{0}, 2}\right)\right)+\bar{\theta}\left(\partial_{G}\left(L_{1,2, \alpha}^{t, \lambda_{0}, 2}\right)\right)
$$

since $\bar{g}$ vanishes on 


$$
\left[\dot{M}_{2, \alpha}^{t, \lambda_{0}}+M_{2,0, \alpha}^{t, \lambda_{0}}+M_{0,2, \alpha}^{t, \lambda_{0}}+\theta_{\lambda_{0}}\left(L_{1,1, \alpha}^{t, \lambda_{0}, 1}\right)\right] / \dot{M}_{2, \alpha}^{t, \lambda_{0}} .
$$

But this is clear from the facts that

$$
\begin{aligned}
& g_{F} \circ \partial_{F}\left(\begin{array}{ccc}
\sigma 1 & (\sigma 2)^{\prime} & (\sigma 3)^{\prime} \\
\sigma 4 & \sigma 5 &
\end{array}\right)=(-1)^{\sigma}-(-1)^{\sigma}=0 \quad \text { and } \\
& g_{G} \circ \partial_{G}\left(\begin{array}{ccc}
\tau 1 & (\tau 2)^{\prime} & (\tau 3)^{\prime} \\
\tau 4 & \tau 5
\end{array}\right)=0
\end{aligned}
$$

for $\sigma, \tau \in \widetilde{S}_{5}$.

We shall show that $h$ is a nonzero linear form. We let;

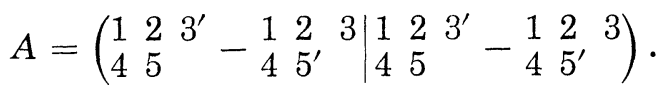

Then $\partial A=0$ and $\bar{g}(A)=1$. This shows that $h$ is non-zero.

(ii) case 2. $t \geq 3$.

We define a linear form $g: L_{1,1, \alpha}^{t, \alpha_{0}, 1} \rightarrow K$ as in (i). We define:

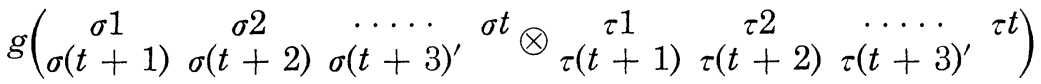

$$
\begin{aligned}
& =\left\{\begin{array}{cl}
(-1)^{\sigma \tau} & \text { (if }\{1, \cdots, t-2\} \subset\{\sigma 1, \cdots, \sigma t\} \cap\{\tau 1, \cdots, \tau t\} \\
0 & \text { (otherwise) }
\end{array}\right.
\end{aligned}
$$

for row-standard bitableaux of shape $\lambda_{0}=(t, 3)$ in $L_{1,1, \alpha}^{t, \lambda_{0}, 1}$. Note that $g$ admits an expression $g=g_{F} \otimes g_{G}$ in an obvious manner as in case (i). It holds that

$$
\begin{aligned}
& g_{F} \circ \square_{\lambda_{0}}^{(t+2,1)}\left(\begin{array}{c}
\sigma 1 \\
(\sigma(t+3))^{\prime}
\end{array}{ }^{\sigma 2 \cdots \sigma(t+2)}\right)=0 \quad \text { and }
\end{aligned}
$$

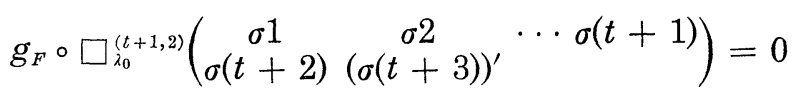

(which can be shown by straightforward computation). Using [5, Lemma I.3.9], it is easy to see that

$$
\begin{aligned}
\operatorname{Im} & \square \lambda_{0} \\
\quad & \cap \wedge^{t} F \otimes \wedge^{2} F \otimes D_{1} F \\
= & \square{\lambda_{0}}^{(t+1,2)}\left(\wedge^{t+1} F \otimes \wedge^{1} F \otimes D_{1} F\right)+\square_{\lambda_{0}}^{(t+2,1)}\left(\wedge^{t+2} F \otimes D_{1} F\right) .
\end{aligned}
$$

Hence, we have $g_{F}$ is zero on $\left[\operatorname{Im} \square_{\imath_{0}} \cap \wedge^{t} F \otimes \wedge^{2} F \otimes D_{1} F\right]_{\alpha(F)}$, where $\alpha(F)$ is the weight $(1,1, \cdots, 1,0,0, \cdots)$. Similarly, we have $g_{G}$ is zero on [Im $\left.\square_{\lambda_{0}} \cap \wedge^{t} G \otimes \wedge^{2} G \otimes D_{1} G\right]_{\alpha(G)}$, where $\alpha(G)$ is also the weight $(1,1, \cdots$, $1,0,0, \cdots)$. Since $\theta_{\lambda}\left(L_{1,1, \alpha}^{t, \lambda, 1}\right)+\dot{M}_{1,1, \alpha}^{t, \lambda}=M_{1,1, \alpha}^{t, \lambda}$ by [5, Lemma IV. 2.2], $g$ induces a linear form $\bar{g}: M_{1,1, \alpha}^{t, \lambda_{0}} / \dot{M}_{1,1, \alpha}^{t, \lambda_{0}} \rightarrow K$, and we extend the definition of $\bar{g}$ as in 
case 1. By an argument similar to the proof in case (i), it is easy to see that $\bar{g}$ induces $h: E \rightarrow K$.

We shall show that $h$ is nonzero. If we put

$$
A=\sum_{\sigma, \tau \in \Im_{t, 3}}(-1)^{\sigma \sigma}\left(\begin{array}{ccccc}
\sigma 1 & \sigma 2 & \cdots \cdots & \sigma t \\
\sigma(t+1) & \sigma(t+2) & \sigma(t+3)^{\prime}
\end{array} \mid \begin{array}{ccc}
\tau 1 & \tau 2 & \cdots \cdots \\
\tau(t+1) & \tau(t+2) & \tau(t+3)^{\prime}
\end{array}\right)
$$

(remember that $\left.\mathfrak{S}_{i, \jmath}=\left\{\sigma \in \mathbb{S}_{i+\jmath} \mid \sigma 1<\cdots<\sigma i, \sigma(i+1)<\cdots<\sigma(i+j)\right\}\right)$ then $\partial A \in \dot{M}^{t, \lambda_{0}}$, and $\bar{g}(A)=\left(\begin{array}{l}5 \\ 3\end{array}\right)^{2}=100 \neq 0$. Hence, we have $h \neq 0$.

By case 1 and case 2 above, we have completed the proof of Theorem 3.1.

Corollary 3.2. The rank of the module $\boldsymbol{X}_{4}^{t}$ does not depend on the characteristic, if and only if $t=1$ or $t \geq \min (m, n)-2$.

Proof. The 'if' part is [5, Corollary IV. 2.12]. Since $\mathscr{I}^{t, t+3}$ is a universally free complex, and $H_{i}\left(\mathscr{I}^{t, t+3}\right)=0$, if $i \neq 2,3$, the rank of $\boldsymbol{X}_{4}^{t}=$ $H_{3}\left(\mathscr{J}^{t, t+3}\right)$ depends on the characteristic if $\operatorname{rank} H_{2}\left(\mathscr{I}^{t, t+3}\right)=\beta_{3, t+3}$ depends on the characteristic. So the 'only if' part follows from the theorem.

Remark 3.3. An argument quite similar to the proof of the theorem shows that $E_{2}^{\infty, 1,(2,1)} \neq 0$, and $E_{2}^{\infty, t,(t, 2)} \neq 0$ for $2 \leq t \leq \min (m, n)-2$, if $R=\boldsymbol{F}_{2}$. It follows that the natural map $H_{2}\left(L^{t,(t+2)}\right) \rightarrow \boldsymbol{X}_{3}^{t}$ is not surjective, if $t \leq \min (m, n)-2$ (even if $t=1$ !) and if $R=\boldsymbol{F}_{2}$. In fact, if we put

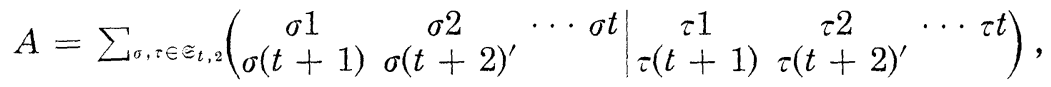

then $\partial A \in \tilde{\mathscr{I}}^{t+1, t+2}$, so $S \pi(A) \in Z_{3}^{\iota}$ (= $Z_{3}^{t, t}$, in the notation of [1]). But $S \pi(A)$ is not contained in the image of $\alpha^{t, t+2}: L^{t,(t+2)} \rightarrow Z_{3}^{t}$. Since $\partial S \pi(A) \in \boldsymbol{X}_{2}^{t+1}$, there exists $B \in \operatorname{Im} \alpha^{t, t+2}$ such that $\partial S \pi(A)=\partial B$, by Kurano's first syzygy theorem. Hence, $S \pi(A)-B \in \boldsymbol{X}_{3}^{t}$, but $S \pi(A)-B \notin \operatorname{Im} H_{2}\left(\alpha^{t, t+2}\right)$.

Therefore, $\boldsymbol{X}_{3}^{t}$ does not have a standard basis as $\boldsymbol{X}_{2}^{t}$ has, although $\boldsymbol{X}_{3}^{\iota}$ is universally free.

Remark 3.4. We have seen that $\boldsymbol{X}^{t}$. is not a universally free $G L(F)$ $\times G L(G)$ complex in the case $2 \leq t \leq \min (m, n)-3$. Recently, the author [4] proved that the Betti numbers of $I_{t}$ are independent of the characteristic in the case $t=1$ or $t \geq \min (m, n)-2$. So $\boldsymbol{X}^{t}$. is universally free in this case, and is the linear part of the resolution. 


\section{REFERENCES}

[1] K. Akin, D. A. Buchsbaum and J. Weyman, Resolutions of determinantal ideals: the submaximal minors, Adv. in Math., 39 (1981), 1-30.

[2] —-, Schur functors and Schur complexes, Adv. in Math., 44 (1982), 207-278.

[ 3 ] J. A. Eagon and D. G. Northcott, Ideals defined by matrices and a certain complex associated with them, Proc. Roy. Soc. Ser. A, 269 (1962), 188-204.

[ 4 ] M. Hashimoto, Resolutions of determinantal ideals: $t$-minors of $(t+2) \times n$ matrices, in preparation.

[5] M. Hashimoto and K. Kurano, Resolutions of Determinantal ideals: $n$-minors of $(n+2)$-square matrices, to appear in Adv. in Math.

[6] K. Kurano, The first syzygies of determinantal ideals, to appear in J. Algebra.

[7] A. Lascoux, Syzygies des variétés déterminantales, Adv. in Math., 30 (1978), 202-237.

[8] P. Pragacz and J. Weyman, Complexes Associated with Trace and Evaluation. Another Approach to Lascoux's Resolution, Adv. in Math., 57 (1985), 163-207.

[9] P. Roberts, "Homological invariants of modules over commutative rings," Les Presses de l'Université de Montreal, Montreal 1980.

Department of Mathematics

School of Science

Nagoya University

Chikusa-ku, Nagoya 464-01

Japan 